\title{
DER WIDERRUF EINES TAUFGESINNTEN IN WESEL AM 26 DECEMBER 1 วั4.
}

DOOR

P. BOCKMÜHX.

In einem Sammelwerk von sorgfältigen Abschriften aus Acten des Kirchen- und Stadtarchivs der Stadt Wesel, welches der Weseler Prediger Antonius van Dorth (16551695) anfertigte und das jetzt im Düsseldorfer Königl. Staatsarchiv ${ }^{1}$ ) sich befindet, wird der Widerruf eines Taufgesinnten, Ludwig van Schoenwinckel aus dem Jahre 1547 mitgeteilt. Obwobl er sich in der Form nicht von ähnlichen Widerrufen wesentlich unterscheidet, ist er doch durch die begleitenden Umstände höchst interessant. Die Begebenheit fällt in die Zeit des Herzogs Wilhelm $V$ von Gülich, Cleve, Berg (1539-1592), der scharfe Massregeln gegen die Taufgesinnten zumal in seiner Abhängigkeit $\nabla$ on Kaiser Karl $\nabla$ in seinen Landen eingeführt hatte.

Zumal in Wesel hatten die Taufgesinnten grosse Verbreitung gefunden und bereits im Jahre 1535 (am 19 Februar und 13 März) hatte der Rat von Wesel, stolz auf seine verbrieften Stadtrechte, zehn taufgesinnte Männer - unter ihnen den Stadtrentmeister Otto Vïnck -, zum Tode verurteilt und hinrichten lassen ${ }^{2}$ ).

1) H. S., A. 50, XVI.

2) Wolters, Zur Kirchengeschichte Wesels, S. 67. 
Was nun den Fall Ludwig van Schoenwinckel angeht, so wissen wir darüber freilich nichts weiteres zu berichten als das, was in der nachstehenden Schilderung des Vorganges anschaulich mitgeteilt wird. Schauplatz desselben ist die Mathena-Kirche in Wesel. Der Prediger, der die Kirchenzucht gegen Ludwig zum Vollzuge brachte, Antonius Mechlinensis, ist hauptsächlich durch das, was Wolters und Lic. Herm. Hamelmann ${ }^{1}$ ) über ibn zu berichten wissen, näher bekannt. Er trug auch den Beinamen : Morenanus, und scheint in Mecheln geboren zu sein. Der erangeliscb gerichtete Weseler Prädicant Imandus Ortzenius Zeelandus hatte ihn im Auftrag des Senates von Wesel dorthin berufen. Bereits beim Osterfest des Jahres 1540 batte er mit Imandus Zeelandus das $h$. Abendmahl unter beiderlei Gestalt an 1500 Bürger der Stadt Wesel ausgeteilt. Am 27 November 1540 wurde er zum Prior des Augustinerklosters in Wesel berufen. Er predigte in der Kapelle dieses Klosters und in der Mathena-Kirche. Zur Zeit des Interims musste er auf Befehl des Kaisers und des Herzogs sein Amt niederlegen und Wesel im Jahre 1548 zum Leidwesen der Weseler Bürgerschaft verlassen. Er ging nach Ostfriesland, wo er im Dienste der Gräfin Anna von Oldenburg tätig war und sich als einen mannhaften Vertreter des evangelischen Glaubens erwies.

Wol war Wesel im Jahre 1547 offiziell noch dem römisch-katholischen Bekenntnisse zugetan, aber reformatorische Anschauungen waren doch schon zur Geltung gekommen und damit der Grundsatz grösserer Duldsamkeit. Man mag aus der Verhandelung gegen Ludwig van Schoenwinckel ersehen, dass man nicht mebr mit Feuer und Schwert gegen irrende Mitchristen loszog, sondern „säuberlich mit dem Knaben Absalom fahren" wollte.

Nun folge der Bericht ${ }^{2}$ ). Er ist in einer Sprache abge-

1) Opp. omn., Lemgoviae 1711, S. 806, 1016, 1017.

2) Düss. Staatsarch., H. S., A. 50, Bd. XVI, S. 17-19. 
fasst, die sich als ein Gemisch aus hoch- und niederdeutschem Idiom darstellt. Er lautet, wie folgt:

\author{
"Lodwich van Schoinwinckel nachfolgender gestalt tho \\ begnadigen.
}

Dat hij den neisten heiligen dach ein lijnen laeken vmbnehme vnd für den Predicăten in der kercken kome, vnd vp einer banck vor den predigstoil sitten gaen rnd wanneer die predig geendigt, sall der Prediger van dem stoel verkündigen, als ferner:

Anfenglich, dat hij bekenn, gegen Gott ynd sein heilig wordt gesundigt te hebben rnd dat hij dairumb in rngeloue gekomen, sijne tijtliche Overicheit hoichlichen vertornet, derhaluen hij billicher wijss in die straif lijfs rnd levens na ordnung des heiligen Römischen Rijx vnd vnses gn. Herrn Edicte gefallen rnd die straif ook schuldig gelick andern tho ontfangen, vnd hierup genoempten Lodwich tho fragen, of hij dat bekent dem also werc vnd darup mit apener 1) stijmmen oen antworten laeten.

Volgens kortlich den gemeinen man tho vermanen, dwijl gemelter Ludwich ein mitbürger der Christenheit, durch ingeven des boesen Vijantz van lantleuffigen "), onnutten predigeren und rechten Antichristen in den erschröcklichen irthumb gefüret vnd darumb von synem jrsall afthostain willich wer, dat hij dan apentlick die artickeln, dae hij in geirret eerst bekent wair tho syn, und darnae oem für tho halden, als folgt:

Of hij dan geloeffe vud hielde, dat Christus Iesus warhaftiger leuendiger Sonn Gots, ron dem heiligen Geist ontfangen rnd vith der gebenedieder reiner Iouffern Marien syn menschliche natuir angenomen vnd gebaren sy, darup Lodwich sall antworden.

Item, of hij geleuft rnd helt, dat in dem hoichwerdigen Sacrament des Altairs ader Aventmails der ware lijff vnd bloet Christi wesentlich vnd gegenwertich sy vnder gestalt des broits vnd wyns lichamlich vitgereicht rnd ontfangen werde. Darup tho antworden.

Item, of hij bekent, ein heilige Doep in der Christlicher gemein vnd dat der Doep, die in der Kyntheit gereicht vad vitgedeilt werde, den waer recht Christliche Doep sy vod dat

1) Lauter, deutlicher.

2) Als ein solcher Landläufer, d. h. umherreisender Prediger, wird ein Herr „Walter" genannt. 
gein wederdoep in der gemeine Christenheit soll gebruickt worden / vnd darup ock tho antworden.

Item, dat hij fast alle anderen jrrungen vnd boesen artickeln, dem Widerdoep rnd Sacrament anhangig, gantzlich afsacht vnd wederspricht, vnd darup tho antworden.

Wanneir dit geschiet, datt oen nochmals sonderling mit naemen rnd thonaemen tho fragen, of hij dat ock secht vith inniger ioestand menschlicher ontsicht ${ }^{1}$ ), of fristungh synes leuens, rnd darumb recht bekent synen gelouen rnd Gott den Herrn noch synen heiligen Geist niet bedriegen vnd hierup gruntlich tho antworden.

Als hij den vpgerurter gestalt sonderlings vnd int gemein geantwort, sal der prediger auermals vermanen.

Nachdem genoemte Lodwich bekent dat hij also tegen Gott vnd syne tytliche Overicheit gesundigt rnd syne negsten geärgert heeft rnd sich des apentlich bekent, dat hij dan dem Almechtigen hoichlich dancke, hij uith der dwalingen geredt, vith den umwech up den rechten weg bracht, rnd van der loege in die warheit, vnd also vith der boesen geselschaft wieder in gemeinschap der Christgelouigen gekommen sy vnd darumb innichlich bitt, dat der Her syner guedig rnd barmhertich sye, rnd soliche syne ouertredung vnd vergetenheit durch sijne grundtlose barmhertigheit vergeuen vnd vertyen wolle rnd folgens dat gemein folck die vurbith mit eln Vader Unser doin laeten.

Wanneir dit geschiet, sall vorgemelte Lodwich mit synem doitkleidt van der banck gain, an den altair ein bernende Kerse offeren vnd van dair wedergain an die banck, vad up syn Knijen ein Vader Unser rnd Engelsche gruit spreken. Vnd wan dit geschiet, weet der Amptmann mijnes $g$. Herrn beuehl na kommen tho Iaten".

(Die Dominica altera a nativitate Christi anno 47 juxta hanc cedulam publice reclamavit ac egit poenitentiam Lodewicus Schoenwinckel, Concionatore Antonio Mechelinensi praelectore.)

Odenkirchen.

1) Rücksicht. 